\title{
Oficinas Terapêuticas Com Música em Saúde Mental
}

\author{
Elisiane Damasceno Marques Nascimento ${ }^{1}$, Vivian Lemes Lobo Bittencourt ${ }^{2}$, \\ Carolina Renz Pretto ${ }^{3}$, Cátia Cristiane Matte Dezord ${ }^{4}$, Sabrina Azevedo Wagner Benetti ${ }^{5}$ \\ Eniva Miladi Fernandes Stumm ${ }^{6}$
}

\begin{abstract}
RESUMO
Objetivo: Discutir sobre a utilização de oficinas terapêuticas com o uso de música para indivíduos em sofrimento psíquico em um Centro de Atenção Psicossocial. Método: Relato de experiência realizado por uma enfermeira e uma psicóloga em um Centro de Atenção Psicossocial com 40 usuários, que participam das oficinas de música juntamente com as profissionais responsáveis. Resultados: O uso da música para fins terapêuticos mostrou-se efetivo. Ocorreram manifestações dos usuários referentes à sensação de bem-estar, raciocínio, expressão corporal, integração com os demais participantes e melhora do vínculo com a equipe técnica. Discussão: Redefinir o objeto de trabalho implica repensar os meios de intervenção, e adotar tecnologias que respondam ao projeto proposto e que possibilitem momentos de intersecção de sujeitos. Essas ações certamente contribuem no sentido de potencializar a interação entre usuário e trabalhador, saberes e representações, de maneira democrática e respeitosa. A música exerce influência psicológica sobre o comportamento do indivíduo. Ela possui a capacidade de reconstruir identidades, integrar pessoas por meio de seu poder de inserção social e redução da ansiedade, melhorar a autoestima, além de funcionar como importante meio de comunicação. Conclusões: A experiência vivenciada promoveu a amplitude do conhecimento acerca das ações e promoção da qualidade de vida dos usuários e do incentivo da capacitação de profissionais técnicos para aperfeiçoar na prática clínica do processo de reabilitação psíquica dos usuários assistidos.
\end{abstract}

Palavras-chave: Serviços de saúde mental. Enfermagem. Música.

\section{THERAPEUTIC OFFICES WITH MUSIC, IN MENTAL HEALTH}

\section{ABSTRACT}

Objective: Discuss therapeutic workshops with the use of music, for individuals in psychological distress, in a Psychosocial Care Center. Method: Experience report performed in by a nurse and a psychologist a Psychosocial Care Center, with 40 users participating in the music workshops, along with the professionals responsible. Results: The use of music for therapeutic purposes was effective. Users' manifestations related to the sensation of well-being, reasoning, corporal expression, integration with the other participants and improvement of the link with the technical team. Discussion: Redefining the work object involves rethinking the means of intervention, adopting technologies that respond to the proposed project and that allow moments of intersection of subjects. These actions certainly contribute to maximize the interaction between user and worker, knowledge and representations, in a democratic and respectful way. Music exerts psychological influence on the behavior of the individual. It has the ability to rebuild identities, integrate people through their power of social insertion and reduction of anxiety, improves self-esteem, and functions as an important means of communication. Conclusions: The lived experience promoted the amplitude of the knowledge about the actions and promotion of the quality of life of the users, and of the incentive of the qualification of technical professionals to improve in the clinical practice of the process of psychic rehabilitation of the assisted users.

Keywords: Mental health services. Nursing. Music.

Recebido em: 15/1/2018

Aceito em: 24/4/2018

\footnotetext{
Enfermeira. Especialista em Enfermagem em Saúde Mental. lisinascimento12@gmail.com

Enfermeira. Mestre em Atenção Integral à Saúde. Docente no curso de Graduação em Enfermagem na Universidade Regional Integrada do Alto Uruguai e das Missões, campus Santo Ângelo. vivillobo@hotmail.com

3 Enfermeira. Mestre em Atenção Integral à Saúde. carol_pretto14@yahoo.com.br

${ }^{4}$ Mestre em Atenção Integral à Saúde. Docente no curso de Graduação em Enfermagem na Universidade Regional do Noroeste do Estado do Rio Grande do Sul (Unijuí). catiacmatte@yahoo.com.br

Enfermeira. Mestre em Atenção Integral à Saúde - Unijuí/Unicruz. Especialista em Gestão e Saúde no Sistema Prisional - UFMG e Gestão em Saúde: Práticas Coletivas - URI. sabrina.benetti@hotmail.com

${ }^{6}$ Enfermeira. Doutora em Ciências-Enfermagem pela Universidade Federal de São Paulo (Unifesp). Docente permanente no Mestrado PPGAIS na Universidade Regional do Noroeste do Estado do Rio Grande do Sul (Unijuí). eniva@unijui.edu.br
} 


\section{INTRODUÇÃO}

No campo da saúde mental, desde o Movimento da Reforma Psiquiátrica Brasileira, tem-se ampliado a participação de usuários em discussões que envolvem questões relativas às políticas em saúde mental, com o intuito de oportunizar ações inovadoras (NETO; AMARANTE, 2013). Assim sendo, cabe ao profissional de saúde que atua nesse cenário o uso de estratégias humanizadas que envolvam equipe, usuários em sofrimento psíquico e família, de maneira a fortalecê-los para o enfrentamento do processo de adoecimento mental.

Os Centros de Atenção Psicossocial (Caps) integram a rede de atenção à saúde e assistem pessoas em sofrimento psíquico e seus familiares, constituindo-se em serviços reguladores da assistência em saúde mental. Oficinas terapêuticas, conforme o modelo assistencial de saúde mental proposto pela Lei no 10.216, de 6 de abril de 2001, constituem-se em uma das formas de tratamento oferecidas nos Caps (BRASIL, 2012). Os profissionais do Caps, por meio de atividades terapêuticas expressivas e oficinas, expandem cotidianamente o uso da arte no processo de cuidar e escutar, por meio da criação de novas possibilidades existenciais para o sujeito (LIMA; VIEIRA; SILVEIRA, 2015).

Os Caps são serviços estratégicos propostos pela reforma psiquiátrica e possuem diretrizes de atendimento com base comunitária (BERNARDI; KANAN, 2015). Constitui-se em um local aberto, que assiste as pessoas pelo Sistema Único de Saúde (SUS), com equipe técnica e integrada, a qual desenvolve atividades por meio de um plano terapêutico individual, aplicado aos usuários. As modalidades de atendimento podem incluir oficinas terapêuticas, as quais englobam artesanato, pintura em tela, jogos, música, atividades físicas, culinária e terapia em grupos.

No espaço das oficinas, a música auxilia na construção ou no resgate de algumas histórias dos usuários em sofrimento psíquico e funciona como disparadora de memórias, contribuindo para o alívio da ansiedade e do estresse, favorecendo a reflexão (FRANZON et al., 2016).

A enfermagem é uma arte que tem por base o cuidado com o outro, em busca de desenvolver um trabalho humanizado e direcionado à prevenção do sofrimento psíquico. Além disso, os resultados deste trabalho podem ser importantes por favorecerem a reflexão sobre os benefícios da música como alternativa na melhora psíquica dos usuários de um Caps. Este estudo tem como objetivo discutir sobre a utilização de oficinas terapêuticas com o uso de música para indivíduos em sofrimento psíquico em um Caps.

\section{METODOLOGIA}

A presente pesquisa é um estudo de natureza descritiva, tipo relato de experiência. É caracterizada como uma pesquisa descritiva em razão de apresentar uma reflexão sobre uma ação, ou um conjunto que aborda uma situação vivenciada no âmbito profissional (CAVALCANTE; LIMA, 2012).

Foram realizadas oficinas com música com 40 usuários de um Caps, com idades entre 40 e 50 anos. As oficinas foram realizadas duas vezes na semana durante dois meses, totalizando 18 encontros de uma hora cada. Os encontros foram conduzidos por uma enfermeira e uma psicóloga.

Conforme o redirecionamento do modelo assistencial de saúde mental proposto pela Lei no 10.216, de 6 de abril de 2001, as oficinas terapêuticas são uma das formas de tratamento oferecidas nos Caps, serviços considerados hoje como reguladores da assistência em saúde mental (BRASIL, 2001). Mediante as atividades terapêuticas expressivas e oficinas, os profissionais do Caps expandem cotidianamente o uso da arte no processo de cuidar, por meio do estabelecimento de novas possibilidades existenciais para o sujeito (TAVARES; SOBRALI, 2005).

Essas oficinas tinham como principal objetivo a promoção de interação e convivência entre os usuários e os profissionais, com a utilização da criatividade artística como atividade e a oferta de um espaço que propicia a troca de experiências. As oficinas terapêuticas propõem à pessoa em tratamento psiquiátrico a sua inserção em ações que possam, com o desenvolver de atividades artísticas e artesanais, ampliar seus meios de comunicação e, assim, recuperar a sua cidadania (RAUTER, 2012).

\section{DESCRIÇÃO DA EXPERIÊNCIA}

A vivência foi realizada em um Caps a partir da organização de oficinas de música coordenadas pela enfermeira e pela psicóloga do serviço. O espaço onde ocorreu as oficinas é amplo e climatizado, com o intuito de proporcionar um ambiente agradável aos participantes. A oficina iniciava com canções e, no decorrer, os usuários eram orientados a tocar algum instrumento. Aqueles que já possuíam conhecimento musical auxiliavam os demais. Buscava-se dar liberda- 
de aos usuários para a escolha das canções e ritmos, para que cada um desenvolvesse estímulos musicais conforme sua condição física e psíquica.

As ações consistiram em atividades artísticas, artesanais, com acesso ao convívio e comunicação, com a finalidade de recuperar sua cidadania. Na execução das oficinas priorizou-se o trabalho com o uso da música. Inicialmente, a oficina era realizada nas manhãs de segunda-feira; gradativamente, com a adesão dos usuários, o número de participantes aumentou e foi necessário ampliar os dias de oferta da oficina de música. Atualmente, são realizadas às segundas e quartas-feiras com 40 participantes em tratamento psiquiátrico; homens e mulheres com média de 45 anos de idade.

Na experiência ora vivenciada nas oficinas propostas, evidencia-se que os usuários participantes demonstraram e referiram bem-estar, melhora do raciocínio, expressão corporal, integração com os demais participantes e aumento do vínculo com a equipe técnica. Durante as oficinas de música, os instrumentos utilizados sempre eram de escolha dos usuários, com certa flexibilidade do coordenador do grupo. Nesse contexto, observou-se que as seleções de instrumentos e meios a serem utilizados nas intervenções musicais baseavam-se na criatividade do profissional que as realizava. Os instrumentos variaram de acordo com o interesse do usuário, preferencialmente instrumentos de cordas, como violão, violino, cavaquinho, teclado e de percussão, ou aparelhos musicais como, CD player e rádio.

A maioria dos participantes da oficina de música apresentou alegria no semblante no horário da mesma. Percebe-se que a relação do profissional com os usuários é fortalecida, facilita o desempenho do trabalho, diminui a ansiedade e sofrimento, no momento em que é executada a atividade, por alguns minutos todos parecem se transportar às letras que as canções expressam.

\section{DISCUSSÃO}

Redefinir o objeto de trabalho implica repensar os meios de intervenção, adotar tecnologias que respondam ao projeto proposto, que rompam com a organização médica do serviço e possibilitem momentos de intersecção de sujeitos. Essas ações certamente contribuem no sentido de potencializar a interação entre usuário e trabalhador, saberes e representações, de maneira democrática e respeitosa (RAUTER, 2012).
A oficina terapêutica na lógica da atenção psicossocial é um modelo inovador para o processo de tratamento da doença mental. Ela permite a projeção de conflitos internos e/ou externos dos sujeitos por meio de atividades artísticas, de maneira a valorizar o potencial criativo, imaginativo e expressivo do usuário (AZEVEDO; MIRANDA, 2011).

Atualmente, a música e seus elementos contribuem no sentido de favorecer a expressão sonora. Para ampliar fronteiras internas são utilizados o som, o ruído, o silêncio, as intensidades em frequências regulares e irregulares, constantes e inconstantes, estáveis e instáveis, definidas e indefinidas, afinadas e desafinadas. A música é um instrumento terapêutico complementar, capaz de promover um ambiente mais tranquilo e acolhedor, além de propiciar relaxamento, entrega e escuta. A utilização da música motiva resultados positivos por não ser realizada isoladamente, mas inserida no planejamento junto a outras medidas terapêuticas, tornando perceptível a contribuição desse recurso (AVELINO et al., 2014).

A música exerce influência psicológica sobre o comportamento do indivíduo. Ela possui a capacidade de reconstruir identidades, integrar pessoas por meio de seu poder de inserção social e redução da ansiedade, melhora a autoestima, além de funcionar como importante meio de comunicação. A música integra elementos históricos, sociais e culturais das pessoas, de modo que é importante considerar a individualidade de cada participante (SILVA et al., 2013).

Oficinas terapêuticas desenvolvidas em uma instituição de saúde proporcionaram um clima agradável, com compartilhamento de sentimentos e com questões relativas ao estado psíquico trabalhadas. A música promove a criação de um ambiente terapêutico, no qual o usuário sente-se valorizado e acolhido, além do sistema convencional de atendimento à saúde. Nesse contexto, a realização de uma oficina de música no ambiente terapêutico no campo da saúde mental proporciona uma gama de respostas. A disposição dos usuários é refletida ou provocada pelos padrões musicais, mediados pelo contexto cultural e pelas experiências anteriores com a música, isto é, pelo fator aprendizagem (RADOCY; BOYLE, 2012).

O Caps deve se constituir em território existencial que possibilite reinventar a vida em seus aspectos diários, pois é no cotidiano que pessoas com transtornos mentais se encontram privadas de liberdade e são nominadas de doentes mentais. As atividades funcionam como catalisadoras existenciais em que os usuários podem reconquistar ou conquistar o seu cotidiano. Quanto às atividades terapêuticas, igualmen- 
te devem se constituir em espaços de produção de subjetividades, com diálogo, interação, reciprocidade e vínculo (RAUTER, 2012).

Observou-se que, no momento em que as canções eram entoadas, os usuários cantavam, dançavam e apresentavam traços de alegria em suas faces, e produziam composições próprias com expressões verbais que ilustravam suas vivências naquele ambiente. A mudança que a música proporciona na vida das pessoas tem sua subjetividade e depende do ponto de vista de cada um e do momento que a pessoa vive; cada um tem sua história, que sempre será única, pessoal e original (MENDES et al., 2015). Pesquisa no Estado de São Paulo, em 80 Caps, mostrou que, em 44 desses centros, ocorrem oficinas de música. As respostas acerca dos estímulos vivenciados nas oficinas foram expressos com emoções, afetos, destreza técnico-musical, integração social e familiar (SALVADOR, 2013).

As oficinas em Saúde Mental podem ser consideradas terapêuticas quando possibilitam aos usuários dos serviços um lugar de fala, expressão e acolhimento. Além disso, avançam no caminho da reabilitação, pois exercem o papel de um dispositivo construtor do paradigma psicossocial. Nesse sentindo, investigações voltadas para os diferentes aspectos dos Caps potencializam subsídios para a instituição ou consolidação da Reforma Psiquiátrica, ao mesmo tempo em que impulsionam um novo território de pesquisa no âmbito das políticas públicas e da avaliação dos serviços de saúde (CASTRO et al., 2013).

Para coordenar um grupo é necessário preparo para atuar em novos modelos de educação em saúde, assumir tarefas e visar sempre à integralidade do cuidado. O grupo espera que o coordenador seja capaz de despertar o interesse dos participantes, considerando os fatores cognitivos e psicológicos do processo de aprendizagem, o que pode ser obtido pela música (SILVA et al., 2013). A experiência de coordenar oficinas com o uso da música direcionada a usuários em sofrimento psíquico foi positiva e instigou a perceber a importância de se construir mais pesquisas em busca de evidências científicas, com o intuito de qualificar a assistência a este porcentual expressivo da população que sofre e que merece ser mais bem cuidada, visando à reintegração social.

\section{CONCLUSÃO}

As oficinas terapêuticas realizadas no Caps vêm ao encontro do preconizado pelos autores, ou seja, elas se construíram em espaços nos quais os usuários em sofrimento psíquico conseguiram se expressar em diferentes formas, seja verbal ou não verbal, e que tiveram como resultado principal a redução do sofrimento vivenciado.

Avalia-se que, no decorrer das oficinas, evidenciou-se aspectos positivos do uso da música, tais como tomadas de decisão quanto à opção por determinados instrumentos, leveza nos movimentos, expressão facial, habilidades técnicas e socialização. Elas cumprem a finalidade de reabilitação psicossocial, promovem ambientes de reconstrução de papéis sociais, intercâmbios e trocas com os espaços sociais externos ao Caps.

Construir este relato foi importante por ampliar conhecimentos sobre essa atividade que vai além e que nos remete à promoção da qualidade de vida dos usuários e que requer da equipe que atua em CAPS capacitação com vistas a aperfeiçoar a prática clínica no processo de reabilitação e reinserção dos usuários na sociedade.

\section{REFERÊNCIAS}

AVELINO, D. C. et al. Trabalho de enfermagem no centro de atenção psicossocial: estresse e estratégias de coping. Revista de Enfermagem da UFSM, Santa Maria, v. 4, n. 4, p. 718-726, 2014.

AZEVEDO, D. M.; MIRANDA, F. A. N. Oficinas terapêuticas como instrumento de reabilitação psicossocial: percepção de familiares. Escola Anna Nery Revista de Enfermagem, São Paulo, v. 15, n. 2, p. 339-345, 2011.

BERNARDI, A. B.; KANAN, L. A. Características dos serviços públicos de saúde mental (Capsi, Capsad, Caps III) do Estado de Santa Catarina. Saúde Debate, Florianópolis, v. 39, n. 107, p. 1.105-1.116, 2015.

BRASIL. Ministério da Saúde. Lei no 10.216, de 6 de abril de 2001. Dispõe sobre a proteção e os direitos das pessoas portadoras de transtornos mentais e redireciona o modelo assistencial em saúde mental. Brasília: Ed. do Ministério da Saúde, 2001.

. Ministério da Saúde. Saúde Mental em Dados, Brasília: Ed. do Ministério da Saúde, 10, ano VII, n. 10, 2012. (Informativo eletrônico). Disponível em: < http://www.consultaesic.cgu.gov.br/busca/dados/Lists/Pedido/Attachments/430410/RESPOSTA_PEDIDO_10_Sade\%20Mental\%20 em\%20Dados\%20dezembro\%202011\%20(2).pdf>.

CASTRO, A. E. S. et al. Análise bibliométrica sobre a inclusão de crianças e adolescentes com deficiência fazendo uso da música como ferramenta central. Revista Ciências de la Salud, Bogotá, v. 11, n. 1, p. 45-58, 2013.

CAVALCANTE, B. L. L.; LIMA, U. T. S. Relato de experiência de uma estudante de enfermagem em um consultório especializado em tratamento de feridas. Journal of Nursing and Health, Pelotas, v. 1, n. 2, p. 94-103, 2012. 
FRANZON, M. A. H. et al. Intervenção musical como estratégia de cuidado de enfermagem à criança com transtorno do espectro do autismo em um centro de atenção psicossocial. Texto contexto enfermagem, Florianópolis, v. 25, n. 1, e.1020025, 2016.

LIMA, D. W. C.; VIEIRA, A. N.; SILVEIRA, L. C. A escuta terapêutica no cuidado clínico de enfermagem em saúde mental. Texto contexto enfermagem, Florianópolis, v. 24, n. 1, p. 154-160, 2015.

MENDES, M. V. S. et al. Crianças com retardo do desenvolvimento neuropsicomotor: musicoterapia promovendo qualidade de vida. Revista Brasileira de Enfermagem, Brasília, v. 68, n. 5, p. 797-802, 2015.

NETO, M. L. A.; AMARANTE, P. D. C. O acompanhamento terapêutico como estratégia de cuidado na atenção psicossocial. Psicologia: Ciência e Profissão, Florianópolis, v. 33, n. 4, p. 964-975, 2013.

RADOCY, R.; BOYLE, D. Psychological foundations of musical behavior. 5. ed. Springfield: Charles C. Thomas Publisher, 2012.

RAUTER, C. Oficinas para quê? Uma proposta ética estética, política para oficinas terapêuticas. In: AMARANTE, P. (Org.). Ensaios: subjetividade, saúde mental, sociedade. Rio de Janeiro: Fiocruz, 2012.

SALVADOR, B. D. Um mapeamento das oficinas de música dos CAPS das regiões de saúde do Estado de São Paulo. Psicologia Fapesp, Unesp, Assis, 2013. Disponível em: <https://www.revistas.unijui.edu.br/index.php/contextoesaude/about/submissions\#authorGuidelines>. Acesso em: 28 dez. 2017.

SILVA, L. B. et al. A utilização da música nas atividades educativas em grupo na Saúde da Família. Revista Latino-Americana de Enfermagem, Ribeirão Preto, v. 21, n. 2, p. 632640, 2013.

TAVARES, C. M. M.; SOBRALI, V. R. S. Avaliação das práticas de cuidar envolvendo arte no âmbito do Centro de Atenção Psicossocial (Caps). Revista Mineira de Enfermagem, Belo Horizonte, v. 9, n. 2, p. 121-125, 2005. 